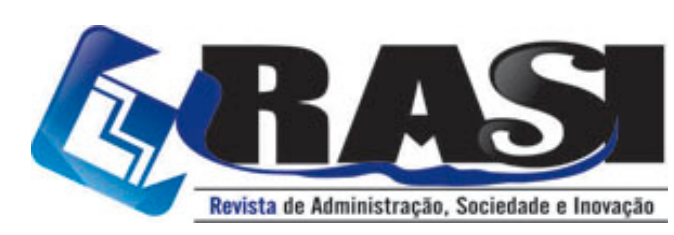

http://www.rasi.vr.uff.br

RASI, Volta Redonda/RJ, v. 7, n. 1, pp. 01-06, Jan./Abr. 2021

Edição Especial em Parceria com a ABEIN

\title{
A Contribuição da Economia Industrial e da Tecnologia Para as Temáticas de Administração, Sociedade e Inovação
}

Janaína Ruffoni, UNISINOS e ABEIN, Editora Convidada

Bruno Brandão Fischer, Editor Associado, FCA/UNICAMP

Marcelo Gonçalves do Amaral, Editor Chefe, PPGA/UFF e PPGDIN/UFF

\section{for unerestate \\ Fluminense}

R. Desembargador Ellis Hermydio Figueira, 783, Bloco A, sl. 218, Aterrado. 27213-415 - Volta Redonda, RJ - Brasil

www.uff.br 


\section{A Contribuição da Economia Industrial e da Tecnologia Para as Temáticas de Administração, Sociedade e Inovação}

$\mathrm{O}$ ano de 2021 se inicia em meio à crise sistêmica instalada e os impactos em toda a sociedade brasileira. Não podemos deixar de desejar votos de um Feliz Ano Novo e um Próspero 2021 mesmo correndo o risco de parecermos levianos. Nunca a busca da prosperidade e da felicidade nos pareceu tão necessária.

Toda a equipe envolvida na RASI tem empreendido esforços em prol da manutenção da missão da revista, que é ser um veículo de difusão do conhecimento. A revista tem sido pouco afetada, pois já se trata de uma atividade com operacionalização remota, exceto pela menor disponibilidade dos revisores. Por outro lado, cada colaborador foi afetado de alguma forma, seja pessoalmente ou vivenciando os efeitos ao seu redor. Nesse sentido, não é possível iniciar este editorial sem novamente nos solidarizarmos com aqueles que estão sendo mais atingidos pela pandemia sanitária, seja diretamente ou pelos seus efeitos econômicos. Nossas preces estão com vocês!

No contexto supracitado, a união de forças se torna um elemento crítico para enfrentar os desafios que se apresentam. Em um momento onde o campo da ciência, tecnologia e inovação (C\&T\&I) é instado a prover soluções e ao mesmo tempo desacreditado pelos governantes, a formação de parcerias, a ampliação de redes e o aprofundamento de laços institucionais surgem como elementos de uma estratégia de fortalecimento conjunto no contexto acadêmico, permitindo manter os avanços científicos, elementos fundamentais para a criação de uma sociedade mais desenvolvida e uma economia pujante (Nelson, 2019; Mokyr, 2004). Desta forma, a RASI tem buscado estabelecer e estreitar relações com diferentes organizações e eventos e dentre diversas iniciativas foi estabelecida uma parceria com a Associação Brasileira de Economia Industrial e Inovação (ABEIN) para a produção de uma edição especial que agora se realiza.

Nesta primeira edição de 2021, certamente outro ano de incertezas e grandes desafios no âmbito da economia e da gestão de organizações públicas e privadas, está sendo apresentada a coletânea de trabalho selecionados em diferentes edições do Encontro Nacional de Economia Industrial e Inovação (ENEI). O ENEI é um encontro acadêmico de âmbito nacional organizado pela ABEIN cuja primeira edição ocorreu em 2016. Para essa Edição Especial, a equipe editorial da RASI, em parceria com a membros da ABEIN, selecionou artigos de alta qualidade, que tratam de temas com contribuições significativas para a inovação e a sociedade brasileira, dando o primeiro passo em uma colaboração que deverá ser estendida por muitos anos e iniciativas.

Fundada em 2015, a ABEIN é uma organização que tem por objetivo desenvolver e promover o conhecimento científico em áreas da Economia Industrial e C\&T\&I. Nesta direção, fornece subsídios para políticas, pesquisas e investigações voltadas ao desenvolvimento econômico e social, promovendo a sedimentação de uma comunidade acadêmica vital para a interpretação crítica dos desafios e direcionamentos do sistema socioeconômico brasileiro. Assim, as quatro edições já realizadas do ENEI forneceram vastos e importantes insumos para discutir questões atreladas à economia e gestão de C\&T\&I no país.

Este editorial, assim como o anterior (Amaral et al., 2020), além de apresentar os artigos que compõem esta edição especial, propõe alguns temas-chave de debate acerca da pertinência de visões envolvendo a perspectiva de economia industrial e da tecnologia e seus 
impactos para a sociedade como um todo. Primeiramente, vale destacar que o Brasil tem como um dos seus principais desafios a saída da incômoda situação de "armadilha de renda média", também conhecida como a situação estrutural do subdesenvolvimento, há bastante discutida por Celso Furtado. Tal condição, composta por flutuações econômicas que não alcançam um ritmo de crescimento que permita a geração sustentada de incremento na renda (Gill et al., 2007) e a realização de processos de catching-up tecnológico, está atrelada à incapacidade do sistema econômico de aprofundar suas bases de conhecimento e se inserir em atividades de alto valor agregado (Agénor, 2016). Ainda que iniciativas de excelência existam no país, as fragilidades em termos agregados são notáveis e persistentes.

Conforme ilustram casos de países que conseguiram efetivamente mudar de patamar em termos de desenvolvimento, tendo, mais recentemente, a Coreia do Sul como exemplo (Kim, 2004), pode-se perceber a centralidade de coordenação em torno das políticas e práticas industriais e de C\&T\&I que levem à avanços tecnológicos (technology upgrading) em setores específicos, bem como dinamização sistêmica através da emergência de empreendedorismo intensivo em conhecimento (Radosevic, \& Yoruk, 2014; 2016). Isto ocorre devido ao esgotamento da capacidade dos países de obterem retornos oriundos de recursos de baixo valor agregado (tipicamente recursos naturais) e de processos imitativos de tecnologias estrangeiras, requerendo, assim, mudanças na estrutura produtiva no sentido de avanços na geração de valor (Perez-Sebastian, 2007; Vandenbussche, et al., 2006). Em termos concretos, isto significa que países em desenvolvimento necessitam aprofundar suas capacidades de aprendizagem e de geração da inovação com vistas a alcançar estágios mais elevados de desenvolvimento (Dahlman, et al., 1987; Lall, 1992; Verspagen, 1991).

No Brasil, apesar de iniciativas pontuais bem sucedidas ao longo das últimas décadas, percebe-se ainda uma falta de continuidade e de estratégias claras nas políticas industriais e de C\&T\&I e na concatenação entre estas políticas com aquelas que abordam o equilíbrio macroeconômico. A estes aspectos devem-se ainda somar estruturas de governança que carecem de coordenação entre diferentes esferas envolvidas. Como resultado, o país permanece, infelizmente, com uma matriz produtiva em processo de desindustrialização, de baixa complexidade e pouco competitiva em termos internacionais (Gala, \& Roncaglia, 2020). E é justamente nesta seara que as abordagens oriundas da economia industrial e da tecnologia podem oferecer perspectivas construtivas para o futuro da ciência, da prática administrativa e da sociedade no Brasil. Vale destacar alguns temas de contribuição neste âmbito:

a. Inserção e evolução das empresas em Cadeias Globais de Valor (CGVs);

b. Condições sistêmicas (ou ecossistêmicas) para o surgimento e dinamização de novas empresas intensivas em conhecimento (de base tecnológica ou não);

c. Diversificação e complexificação das atividades produtivas e industriais no país;

d. Processos de produção, transferência e difusão de conhecimentos e de novas tecnologias, envolvendo não somente empresas, mas também universidades, governo e a sociedade;

e. Análise de instrumentos de fomento à oferta e à demanda de inovações;

f. Políticas que fomentem no curto, médio e longo prazos a inclusão dos Objetivos do Desenvolvimento Sustentável como metas para o sistema econômico.

Tais discussões envolvem necessariamente abordagens complementares que dediquem atenção aos âmbitos micro (firmas e gestão), meso (aspectos setoriais e intersetoriais) e macro 
(políticas e ambiente institucional, econômico e social). Estas diretrizes, evidentemente, não trazem consigo propostas simples de solução e dependem, em grande margem, da qualidade das contribuições teóricas e empíricas que pesquisas relacionadas possam oferecer. Adicionalmente, além da contribuição teórica, espera-se que tais discussões apoiem o caminhar para a construção de um grande acordo nacional em prol do desenvolvimento econômico e social, de forma sustentável, que independente de matizes políticas, seja sustentado por uma agenda comum e consensual dos inúmeros atores da nossa sociedade.

Por fim, espera-se que seja útil a contribuição dos artigos incluídos nesta edição especial da RASI como insumos para enriquecer este debate. Espera-se que tais aportes possam estimular trabalhos em áreas correlatas, ampliando e qualificando estas discussões no contexto nacional. O conjunto de trabalhos discute assuntos diversos e conectados com os desafios discutidos. As temáticas vão desde empresas spin-offs de empregados e empresas startups até redes colaborativas tendo como base a inovação em diferentes contextos passando por estudos em setores chave como as indústrias de biotecnologia e farmacêutica.

\section{Apresentação desta Edição}

Após a introdução a este debate, direcionamos nossa atenção à apresentação do número que inicia o volume 7 , correspondente ao ano de 2021. A partir de um conjunto diverso de artigos publicados nas diferentes edições do ENEI, foram pré-selecionados 52 trabalhos de acordo com as temáticas aderentes ao propósito da RASI e que apresentavam elevada qualidade acadêmica. Os autores interessados em publicar na Revista submeteram seus trabalhos ao processo de avaliação usual do periódico (no qual agradecemos a ABEIN pela sugestão de avaliadores). Na segunda etapa dez trabalhos seguiram no processo de avaliação e o resultado final está, então, apresentado nesta edição especial com a publicação de seis artigos.

A edição é aberta com o artigo do autor Thiego Gonçalves Ferreira, do Instituto Brasileiro de Geografia e Estatística (IBGE), intitulado "Sobrevivência e Crescimento de Spin-off de Empregados no Brasil: Nascimento por Oportunidade ou Necessidade". O trabalho realiza uma análise de spin-offs de empregados e a taxa de sobrevivência destes negócios em relação a outras firmas entrantes com base em informações da Relação Anual de Informações Sociais (RAIS) para o período de 2006 a 2015. O trabalho traz uma contribuição significativa de avançar em uma temática até então pouco explorada.

O segundo artigo intitulado "Mudanças Climáticas como um Fator Exógeno que afeta o Comportamento de Busca Tecnológica", de autoria de Paulo Feitosa, da Universidade de São Paulo (USP), estabeleceu um diagnóstico do processo de mudanças climáticas globais a partir de algum fator exógeno que modifica o comportamento de busca tecnológica das firmas. Para tal, a análise buscou informações na literatura em três níveis principais, da firma, do mercado, e do sistema.

Já o terceiro artigo realiza uma análise da rede de colaboração científica da biomassa da cana-de-açúcar, analisando a formação de clusters de publicações indexadas no banco de dados da Web of Science. Intitulado "An Analysis of Collaboration Networks in Bioenergy: Using the 'Bioen Program' to Evaluate Sugarcane Ethanol Biomass" (em português Análise de Redes de Colaboração em Bioenergia: Utilizando o 'Programa Bioen' para Avaliar Biomassa de Etanol de Cana). Foi escrito por quatro pesquisadores, Carolina da Silveira Bueno e José Maria Ferreira Jardim da Silveira, da Universidade Estadual de Campinas 
(UNICAMP), Roney Fraga Souza, da Universidade Federal de Mato Grosso (UFMT), e Jaim José da Silva Junior, da Universidade de Brasília (UNB).

O quarto artigo desta edição, desenvolvido por Cíntia Siqueira Araújo Soares, pesquisadora do Instituto Federal de Minas Gerais (IFMG), além de três pesquisadores da Universidade Federal de Minas Gerais (UFMG), Allan Claudius Queiroz Barbosa, Bárbara Graciano de Oliveira, Cristina Luiza Ramos da Fonseca (também pesquisadora da Fundação Oswaldo Cruz - FIOCRUZ) tem como título "Um Retrato das Startups de uma Aceleradora Localizada em Belo Horizonte, Minas Gerais". A pesquisa caracteriza as startups existentes no ecossistema de inovação de Belo Horizonte, considerando as pertencentes a um programa de aceleração. Para tanto, foi desenvolvido um estudo de caso com enfoque no Programa de Aceleração da Techmall S.A.

Um grupo de pesquisadores da Universidade Estadual de Campinas (UNICAMP), Caroline Giusti Araújo, Caroline Miranda Brandão, Guilherme Nascimento Gomes e Antônio Carlos Diegues, desenvolveram o artigo "The End of Cheap China? Uma Análise do Catching up Produtivo e da Sofisticação das Exportações Chinesas entre 2001 e 2017”. O artigo buscou avaliar como as mudanças na estrutura produtiva chinesa impactaram os preços de bens industriais exportados pelo país entre 2001 e 2017. Este trabalho tem uma contribuição significativa ao desmistificar o desenvolvimento chinês e trazer possíveis contribuições à discussão nacional.

O sexto artigo, elaborado por pesquisadoras da Universidade Federal de Minas Gerais (UFMG), Luiza Pinheiro, Márcia Siqueira Rapini, e da Universidade Federal do Rio de Janeiro (UFRJ), Julia Paranhos, faz uma análise do grau de incerteza dos projetos do setor farmacêutico. Com o título "Subvenção à Inovação no Setor Farmacêutico Brasileiro: Uma Análise a Partir do Nível de Incerteza", o trabalho é uma analise dos recursos de subvenção econômica providos pela FINEP no período entre 2007 e 2014, buscando descobrir a importância de políticas industriais sistêmicas que incentivem que as empresas brasileiras farmacêuticas no desenvolvimento de atividades de inovação.

A edição se encerra com o artigo elaborado pelos pesquisadores Sarah Cristina Ferreira e Germano Mendes de Paula, da Universidade Federal de Uberlândia, que analisa os impactos da Indústria 4.0 no setor de papel e celulose. A metodologia aplicada consiste na identificação dos efeitos de oito clusters tecnológicos (produção inteligente e conectada, inteligência artificial, tecnologias de redes, biotecnologia e bioprocessos, nanotecnologia, materiais avançados e armazenamento de energia) sobre o setor, a partir da aplicação de questionários para a identificação da influência dessas inovações sobre a realidade mundial e brasileira.

A equipe editorial da RASI e a ABEIN desejam uma boa leitura e um ano de saúde, paz e prosperidade para todos!!

Volta Redonda, $1^{\mathrm{o}}$ de Janeiro de 2021.

\section{Referências}

Agénor, P. (2016). Caught in the Middle? The Economics of Middle-Income Traps. Journal of Economic Surveys, 31(3), 771-791.

Amaral, M. G., Fisher, B. B., Carvalho, R. S., Oliveira, V. C. S., Silva, S. S., \& Carraro, E. R. (2020). A Crise e a Oportunidade da Pandemia - Por uma Nova Política de 
Desenvolvimento. Revista de Administração, Sociedade e Inovação, 6(3), 1-6. https://doi.org/10.20401/rasi.6.3.519

Dahlman, C., Ross-Larson, B., \& Westphal, L. (1987). Managing Technological Development: Lessons from the Newly Industrializing Countries. World Development, 15(6), 759-775.

Gala, P., \& Roncaglia, A. (2020). Brasil, uma Economia que não Aprende: Novas Perspectivas para Entender o nosso Fracasso. São Paulo: Edição do Autor, 243 p.

Gill, I., Kharas, H., Bhattasali, D., Brahmbhatt M., Datt, G., Haddad, M., Mountfield, E., Tatucu, R., \& Vostroknutova, E. (2007). An East Asian Renaissance: Ideas for Economic Growth. Washington, DC: The World Bank.

Kim, L. (2004). The Multifaceted Evolution of Korean Technological Capabilities and its Implications for Contemporary Policy. Oxford Development Studies, 32(3), 341-363.

Lall, S. (1992). Technological Capabilities and Industrialization. World Development, 20(2), 165-186.

Mokyr, J. (2004). The Gifts of Athena: Historical Origins of the Knowledge Economy. Princeton: Princeton University Press.

Nelson, R. R. (2019). On Technological Capabilities and their Acquisition. Science and technology: Lessons for development policy, 71-80.

Perez-Sebastian, F. (2007). Public Support to Innovation and Imitation in a Non-Scale Growth Model. Journal of Economic Dynamics and Control, 31(12), 3791-3821.

Radosevic, S., \& Yoruk, E. (2016). Why do we need a Theory and Metrics of Technology Upgrading? Asian Journal of Technology Innovation, 24(1), 8-32.

Radosevic, S., \& Yoruk, E. (2014). Are there Global Shifts in the World Science Base? Analysing the Catching up and Falling behind of World Regions. Scientometrics, 101(3), 1897-1924.

Vandenbussche, J., Aghion, P., \& Meghir, C. (2006). Growth, Distance to Frontier and Composition of Human Capital. Journal of Economic Growth, 11(2), 97-127.

Verspagen, B. (1991). A New Empirical Approach to Catching up or Falling Behind. Structural Change and Economic Dynamics, 2(2), 359-380. 